\title{
Herpes Simplex Virus Induces the Early Activation of NK Cell via MyD88- Dependent Signal
}

\author{
Stacie N. Woolard ${ }^{1}$, Divya G. Venkat ${ }^{1}$, Evelyn Kurt-Jones ${ }^{2}$ and Uday Kumaraguru ${ }^{*}{ }^{1}$ \\ ${ }^{I}$ Department of Microbiology, James H. Quillen College of Medicine, East Tennessee State University, Johnson City, TN \\ 37614, USA \\ ${ }^{2}$ Division of Infectious Diseases and Immunity, Dept. of Medicine, U Mass Medical School, Worcester, MA 01655, USA
}

\begin{abstract}
In the course of a Herpes Simples Virus type-1 (HSV-1) infection, the bidirectional cross-talk between Natural Killer (NK) cells and Dendritic Cells (DCs) is imperative in mounting an efficient immunological response. Enhanced DC maturation by Toll-Like Receptor (TLR) ligands is considered as a pivotal link between innate and adaptive immune responses, yet the impact of direct TLR signaling on NK:DC interaction during an HSV infection has yet to be examined. We demonstrate that HSV mediates the activation of NK cells and the induction of the cooperative relationship between NK cells and DCs via TLR. During the course of an HSV infection, the initial response required both types of innate cells and demonstrated phenotypic and functional activation much like that of TLR-stimulated cells. Using a MyD88 knock-out system diminished the early interaction between DCs and NK after HSV exposure. Our results indicate that HSV TLRligands modulate the early NK: DC interaction and may be a mechanism used by these innate immune cells to overcome virus mediated immune evasion and the initiation of a potent acquired immune response.
\end{abstract}

Keywords: NK cell, dendritic cells, HSV, TLR.

\section{INTRODUCTION}

Interaction of innate immune cells is essential for inducing and sustaining an immunological response to both pathogen and tumor, especially dendritic cells (DCs) and Natural Killer (NK) cells. In lymphoid organs, DCs exist in two functionally and phenotypically distinct states: immature and mature. Immature DCs (iDCs) are proficient at endocytosis and antigen processing; however, MHC class I surface levels and expression of co-stimulatory molecules are low on iDCs. Therefore, iDCs are proficient in sampling their environment and taking up antigen, yet their ability to present antigens to $T$ cells is deficient [1]. Upon the detection of microbial products via Toll-like Receptors (TLR) or in response to pro-inflammatory cytokines (IL-12, IL-15, IL-18, Type I interferons), iDCs undergo a maturation process creating efficient $\mathrm{T}$ cell priming DCs [2].

NK cells have long been considered important in initiating an immune response [3]. While NK cell activation occurs due to lack of surface MHC class I on targets, target lysis requires a multitude of signals that include stress proteins, cytokines and involvement of other sentinel cells. Although NK cells were originally thought to act independently, accumulating evidence indicates that NK cells also respond to stimuli from other immune effectors, especially DC. Kassim et al. have demonstrated that in vivo ablation of CD11c-positive DCs increase the susceptibility to HSV-1 infection, likely through decreased $\mathrm{NK}$ and $\mathrm{T}$ cell response [4]. Furthermore, DCs are required for most

*Address correspondence to this author at the Department of Microbiology, Box -70579, Quillen College of Medicine, ETSU, Johnson City, TN-37614, USA; Tel: 423-439-6227; Fax: 423-439-8044; E-mail: kumaragu@etsu.edu advantageous activation of NK cell function post primary infection [5].

DCs are present in tissues that HSV initially infects (skin or mucosa). HSV has not only evolved to infect DCs but has also acquired efficient means to prevent DC activation [6]. Although, this process has been characterized as an HSV immune avoidance mechanism, we hypothesize that HSV infected DCs are now capable of productive interaction with NK cells, due to reduced expression of MHC class I molecules combined with the production of IFN- $\alpha$ and IL12, which stimulates NK cells [6].

In a recent report by our group, NK cells were demonstrated to be novel helpers in an anti-HSV adaptive immune response [7]. The quantity and quality of anti-HSV $\mathrm{CD} 8+\mathrm{T}$ cells were diminished in the absence of NK cells. Consequently, mice that lacked NK cells were more susceptible to HSV induced lesions. NK cell supplementation enhanced the function of $\mathrm{CD} 8+\mathrm{T}$ cells; compensated for the loss of CD4+ helper T cells; and were able to partially rescue dysfunctional CTLs that were generated in the absence of CD4+ helper T cells. However, the exact reason for such an influence by NK cells on immune induction is not completely understood. The mechanism of HSV's ability to directly activate NK cells and influence NK:DC interaction remains unknown.

In the following study, we demonstrate that HSV PAMPS influence the bidirectional cross-talk between NK cells and DCs. With both NK cells and DCs expressing TLRs, it is our hypothesis that HSV mediates the early interaction and signaling events of NK cells and DCs via direct TLR engagement. To address HSV PAMP mediated cross-talk initiation, DCs and NK cells were stimulated with UV-inactivated HSV or TLR-ligands to examine function 
and activation. Despite the decreased expression of costimulatory molecules, HSV stimulated dendritic cells significantly augmented the production of DC derived IL-12 and increased $\mathrm{NK}$ activation and cytokine production. To specifically address the role of direct activation by HSV through TLR, DCs and NK cells from MyD88 ${ }^{-/}$were used to examine whether or not activation is dependent upon MyD88 signaling. Our results indicate the possibility that viral TLRligands modulate $\mathrm{NK}: \mathrm{DC}$ interaction resulting in the initiation of a potent acquired immune response.

\section{MATERIALS AND METHODS}

\subsection{Mice}

Female C57BL/6 (7-8 wk old), purchased from Harlan Sprague Dawley, Indianapolis, Indiana, and female transgenic PK136, in which transgenic B cells make PK136 monoclonal antibody to deplete NK cells, from Dr. Yuan, UT Southwest Medical Center (previously described [8]; and HSV-gB transgenic mice, with TCR specific for HSV immunodominant peptide, SSEIFARL, were received from Dr. Francis Carbone, University of Melbourne, Australia [9]. All mice were housed under standard conditions with procedures approved by Institutional Division of Laboratory Animal Research. Animals were kept in specific pathogenfree conditions in the Division of Animal Resources, College of Medicine, East Tennessee State University, which is fully accredited by the American Association for Accreditation of Laboratory Animal Care.

\subsection{Cell Isolation}

Natural Killer cells were isolated from wild-type fresh splenocytes using IMag NK cell enrichment (negative selection) magnetic bead separation techniques. Dendritic Cells were isolated from wild-type splenocytes via DC IMag enrichment selection post collagenase digestion. HSV-gB CD8+ $\mathrm{T}$ cells were isolated from HSV-gB transgenic splenocytes via a CD8 enrichment kit. All cell populations were isolate as per manufacturer's protocols (BD Biosciences) and purity verified via flow cytometry, ( $>95 \%)$.

\subsection{Generation of Bone-Marrow Derived Dendritic Cells (DCs)}

C57BL/6 mice and MyD88 $8^{-/-}$mice were sacrificed and femur and tibia were extracted. Extraneous tissue was removed, and using a $1 \mathrm{ml}$ syringe, the marrow was expelled and placed in a 1:1 solution of Hanks Balance Salt Solution (HBSS-HyClone) and RPMI (HyClone) supplemented with $10 \%$ FBS. The marrow was teased through a cell strainer and a single cell suspension was prepared in $10 \%$ FBS supplemented RPMI. Cells were cultured at $1 \times 10^{7}$ cell/ml in 24-well plate and supplemented with10ng/ml Granulocytic Macrophage-Colony Stimulating Factor (GMSFC) + IL-4 in RMPI. The GM-CSF/IL-4/RPMI was replaced every 48 hours for 5 days.

\subsection{Generation of Bone-Marrow Derived Natural Killer Cells (NKs)}

Using the protocol in 2.3, NK cells were generated from C57BL $/ 6$ mice and MyD88 $8^{-/-}$mice bone marrow. Cells were cultured at $1 \times 10^{7}$ cell $/ \mathrm{ml}$ in a 24 -well plate and supplemented with $50 \mu 1 / \mathrm{ml}$ with T-STIM (BD bioscience) culture supernatant, in $500 \mu \mathrm{l}$ of fresh RMPI $10 \%$ FBS. The TStim/RPMI was replaced every 48 hours for 5 days $[10,11]$.

\subsection{Virus}

HSV-1 KOS and HSV-1 17 strains of viruses were titrated after growth on Vero (CCL81; American Type Culture Collection, Manassas, VA) cell lines. The virus was stored in aliquots at -80 until required.

\subsection{HSV Infection and Stimulation}

In vitro: NK cells and DCs were incubated with UVinactivated HSV-kos (3 MOI before inactivation) for 18 hours at $37^{\circ} \mathrm{C}$ for stimulation experiments. Mock stimulation with diluent (endotoxin free buffer) was used as control.

In vivo: $20 \mu \mathrm{l}$ of HSV-1 17 containing $1 \times 10^{5} \mathrm{PFU}$ of virus was administered via the intra-peritoneal route of infection. All animal experiments were performed in agreement with American Association for Accreditation of Laboratory Animal Care.

\subsection{TLR Ligand Stimulation}

Pre-titrated synthetic ligands for TLR2 (palmitoyl-

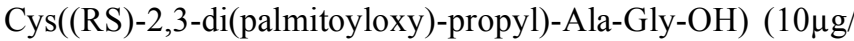
$\mu 1$ ); TLR3 (Polyriboinosinic polyribocytidylic acid

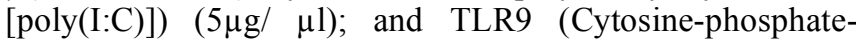
guanine containing oligodeoxynucleotides (CpG ODN) $(10 \mu \mathrm{g} / \mu \mathrm{l})$; were added as a cocktail to cell suspensions seeded at $1 \times 10^{6}$ cells/dose for 18 hours at $37^{\circ} \mathrm{C} @ 5 \% \mathrm{CO}_{2}$. (TLR Ligands were purchased from Invivogen, SD, CA).

\subsection{Co-Culturing Experiments}

Isolated and purified DCs and NK cells were infected or cultured with appropriate TLR ligand stimulation for 18 hours and then co-cultured in $1: 1$ ratio for 18 hours at $37^{\circ} \mathrm{C}$ in $2 \mathrm{ml}$ of RPMI containing $10 \%$ FBS-RPMI to examine phenotypic and functional changes.

\subsection{Cell Surface Staining}

One million splenocytes were incubated with appropriate cell surface markers for 20 minutes. Dendritic cells were analyzed using: anti-CD11c (PE, APC), anti-CD11b (FITC), anti-MHC-I (FITC), anti-MHC-II (PE), anti-CD86 (PE), and anti-CD80 (FITC). NK cells were stained with anti- NK1.1 (FITC, PE), anti-CD3 (FITC, PE, PE-Cy-7, APC), CD69 (FITC). Appropriate isotype antibodies were used as control. All antibodies were purchased from BD biosciences.

\subsection{Intracellular Cytokine Staining}

One million splenocytes were incubated with brefeldin A for 4-5 hours. After incubation, cells were stained with cell surface markers, fixed and permeabilized using perm-fix and perm wash, and, subsequently, stained for intracellular markers with anti-IFN- $\gamma($ PE-Cy7) or IL-12 (APC). All antibodies were purchased from BD Bioscience.

\subsection{In Vitro Proliferation Assay}

NK cells and HSV-gB CD8+ T cells in independent experiments were resuspended to $1 \times 10^{6}$ cells $/ \mathrm{ml}$ in 1xPhospate-Buffered Saline, pH7.2 (PBS). To measure induced proliferation, the cells were labeled with $5 \mu \mathrm{M}$ carboxyfluorescein diacetate, succinimidyl ester (CFSE) 
(Molecular Probes). Post labeling, cells were incubated for 8 minutes at $37^{\circ} \mathrm{C}$ and the reaction was quenched by adding equal volumes of cold fetal bovine serum. The cells were thoroughly washed twice with $1 \times$ PBS avoiding light. Proliferation was measured by examining CFSE dilutions using a flow cytometer.

\subsection{Flow Cytometry}

The staining of cells was performed according to standard protocols and flow cytometric analysis was performed using a four color FACSCalibur4 ${ }^{\mathrm{TM}}$ cytometer. Approximately 100,000 live events were collected. The data were analyzed using Cellquest or FCS Express software.

\subsection{Statistical Analysis}

Graph Pad Prism 4 software was used. Student t-tests were used to determine the statistical significance. To compare samples in co-culture experiments, an analysis of variance (ANOVA) was used to determine significance followed by a Tukey's post-hoc $t$ test. Significance, indicated by $(*)$, represent $\mathrm{p}<0.05$.

\section{RESULTS}

NK cells play a role in shaping the acquired immune response in part by their interaction with DCs. Since, HSV is known to impact the function of both DC and NK cells [6, 12]; we examined the activation of NK cells and induction of NK-DC interaction in response to HSV exposure. This study explores the early events that occur immediately post virus exposure and the involvement of MyD88-dependent (and likely TLR-dependent) signals.

\subsection{HSV Effects on DCs Results in NK Activation}

HSV infection induces the accumulation of DCs and NK cells in the DLN, but the molecular mechanisms subsequent to this process are still not understood [13]. We examined HSV induced changes in DCs that could possibly affect the NK cell population. DCs isolated from C57BL/6 (WT) mice were stimulated with either a cocktail of TLR ligands (TLRL) (PamCys, Poly I:C, and CpG), serving as positive control, or UV-inactivated HSV and then analyzed for co-stimulatory molecule, CD80 (B7.1) and CD86 (B7.2), and MHC-Class I expression. Flow cytometric analysis of CD11 ${ }^{+} \mathrm{CD} 11 \mathrm{~b}^{+} \mathrm{DC}$ revealed a two-fold decreased expression of both costimulatory molecules and surface expression of MHC-I when exposed to HSV in comparison to TLR-L stimulation (Fig. 1A). When compared to control, HSV exposed DC showed increased levels of CD80, CD86, and MHC-Class I, yet HSV has been demonstrated to inhibit DC maturation [6]. To investigate the role of HSV-DCs in NK cell activation, DCs and NK cells were enriched from C57BL/6 splenocytes and co-cultured in 1:1 ratio. DCs were exposed to UV-HSV or stimulated with TLR-L cocktail or unstimulated as described in previous experiments. NK cells were labeled with $5 \mu \mathrm{M}$ CFSE to observe the proliferation induced by co-cultured TLR-DCs or HSV-DCs. Unstimulated DCs and NK cells alone were used as control. Simultaneously, NK cells were also assessed for the capacity to produce IFN $\gamma$ via intracellular staining for flow cytometry. Positive results were recorded for cells expressing CD3NK1.1+ CFSE+. As indicated in Fig. (1B), HSV-DC's ability to activate NK cells was two times greater than the level seen with TLR ligand stimulated DC (TLR-DC) and 12 more than the control (unstimulated DCs). Activation was measured in terms of IFN $\gamma$ production. The percentage of IFN $\gamma^{+} \mathrm{NK}$ cells were $87 \pm 2.5 \%$ in HSV-DCs, while it was $35.8 \pm 2.6 \%$ in TLR-DCs. The control unstimulated DCs were able to induce IFN $\gamma$ in $6.8 \pm 1 \%$ of NK cells. The difference in NK cell stimulating ability of various DCs were statistically significant (HSV-DCs vs TLR-DCs $=\mathrm{P}<0.05$; HSV-DCs $v s$ Control DCs $=\mathrm{P}<0.0001)$. Induction of at least four rounds of NK cell divisions by HSV-DCs was measured. This was significantly greater compared to the NK cells stimulated by TLR-DC which underwent only two rounds of cell divisions. Despite the previously reported negative effects of HSV on DCs, the data presented here demonstrated that as a consequence of HSV exposure, DCs probably because of their lower levels of MHC-I induced NK cell activation and proliferation indicating a possible host mechanism to overcome HSV mediated immune evasion.

\subsection{NK Cells Contribute to Efficient HSV-gB T Cell Priming}

The requirement of DCs for $\mathrm{T}$ cell activation has been demonstrated by many groups; however, the contribution of NK cells in DC mediated activation of T cell has not been appreciated in the context of HSV infection. DCs were isolated from previously infected $\left(1 \times 10^{6} \mathrm{pfu} / \mathrm{mouse}\right) \mathrm{WT}$ and PK136 transgenic mice (which lack NK cells), and subsequently co-cultured with CFSE $(5 \mu \mathrm{M})$ labeled HSV-gB $\mathrm{CD} 8+\mathrm{T}$ cells, obtained from HSV-gB transgenic mice $[8,9]$. DCs and T cells $(0.5: 1.0)$ were co-cultured and then analyzed for HSV specific $\mathrm{T}$ cell proliferation. DCs obtained from infected WT mice increase proliferation of $\mathrm{T}$ cells two log fold $(\mathrm{p}<0.05)$; however, DCs isolated from infected mice lacking NK cells (PK136) were inefficient stimulators of $\mathrm{T}$ cell proliferation (Fig. 2). Taken together, these results reveal that NK cells are required for efficient $\mathrm{T}$ cell priming by DCs with lasting impact on the adaptive immune response. This could very well explain the reason for inferior anti-HSV response in the absence of NK cells as observed by us earlier [7].

3.3. Concomitant Infection of DCs and NK Cells Leads to Increased Phenotypic and Functional Activation

\subsubsection{Effects on DCs}

The individual response of DCs to treatment with TLR-L or HSV has been previously examined, yet the impact on DCs and NK cells interaction and bidirectional cooperation has yet to be explored. DCs and NK cells were isolated from WT splenocytes and purified via magnetic negative selection; subsequently, NK cells were stimulated with pretitrated synthetic TLR-L or exposed to UV-HSV and cocultured with DCs to analyze phenotypic and functional variations.

Phenotypic activation of DCs was determined by measuring the surface expression of co-stimulatory molecule, CD80, and expression of MHC class II; functional activation was determined by measuring the production of IL-12. HSV infection of DCs alone increased the expression of activation markers, CD80 and MHCII; however, the 
(A)

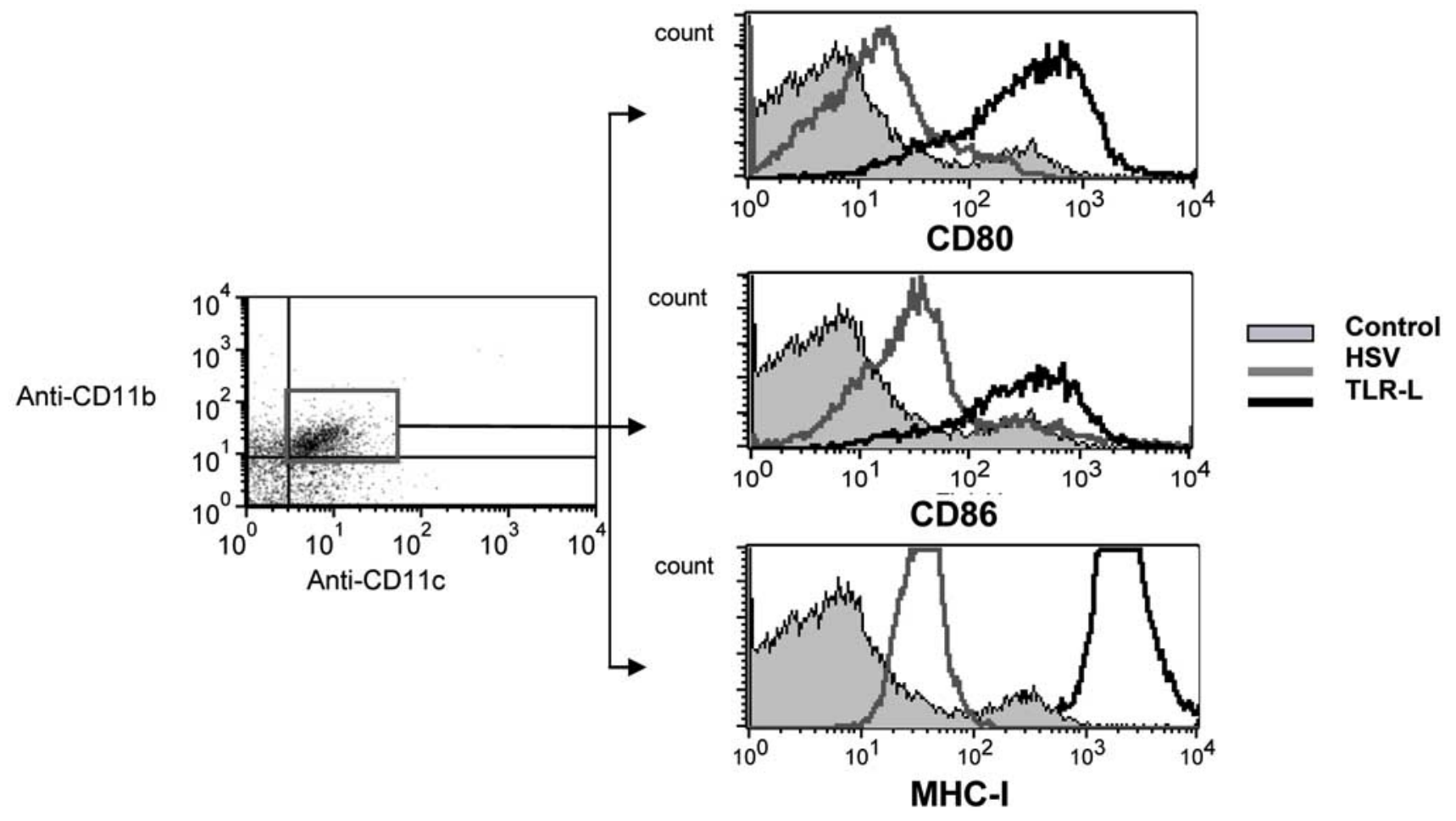

(B)

Unstim. DC

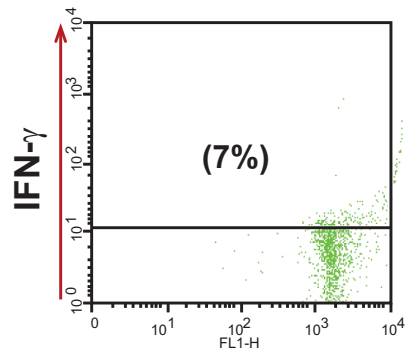

TLR-DC

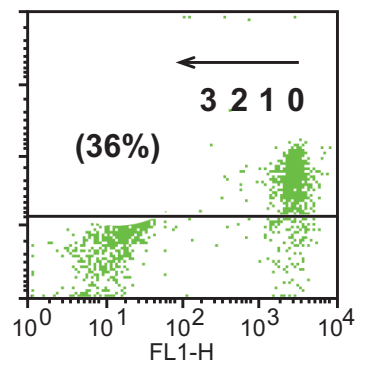

HSV-DC

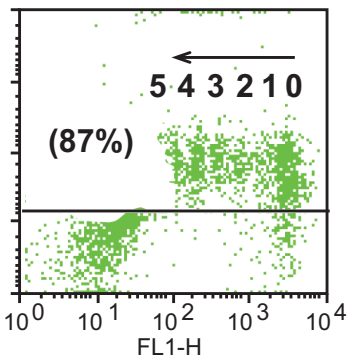

NK-CFSE

Fig. (1). HSV induced negative effect on DCs increases its NK stimulatory ability. (A): HSV Exposed Dendritic Cells show diminished co-stimulatory molecules and MHC- I expression: Dendritic Cells were isolated from C57BL/6 mice and enriched. They were then stimulated with a cocktail of TLR-ligand or $1 \mu 1$ of HSV-kos (3 MOI before UV-inactivation) for 24 hours. Co-stimulatory molecules (CD80-FITC \& CD86-PE) and MHC-I (PE) expression was analyzed using flow cytometry by gating on CD11c+ population. The histogram plot shows the data collected from one of the three experiments. The filled area depicts mock stimulated DCs. (B): HSV stimulation enables DCs to induce NK cell proliferation and cytokine production: NK cells and DCs were isolated and purified using appropriate magnetic beads from age and sex-matched naive C57BL/6 splenocytes. NK cells were labeled with $5 \mu \mathrm{M}$ of CFSE and co-cultured with stimulated (TLR ligand [TLR-Dc] or HSV [HSV-DC]) DCs for 24 hours to examine the impact of HSV- DC on NK cell proliferation by CFSE dilution and cytokine production (IFN- $\gamma$-PECy7). IFN- $\gamma$ production was assayed through standard intracellular staining. Analysis is a representation of CFSE ${ }^{+} \mathrm{IFN}-\gamma^{+} \mathrm{NK}$ cells from one of the experiment. The experiment was repeated at least three times with similar outcome. The number in parenthesis is the percentage of NK cells positive for IFN- $\gamma$ and the numbers below the arrow indicate the rounds of NK cell division. The difference in NK cell stimulating ability of various DCs were statistically significant (HSV-DCs $v s$ TLR-DCs $=$ P $<0.05$; HSV-DCs $v s$ Control DCs $=\mathrm{P}<0.0001)$.

co-infection and co-culture of DCs and NK cells resulted in more efficient upregulation of phenotypic activation markers (results not shown). The pattern was consistent with many other studies. In contrast to phenotypic activation, a significant increase in IL-12 production by DCs was observed in the presence of HSV exposed NK cells. DC function was measured in terms of IL-12 (p70) production, since IL-12 is a critical component in NK cell stimulation. HSV exposure of DCs alone and in co-cultures with NK cells resulted in an increased secretion of IL-12 (Fig. 3). To 


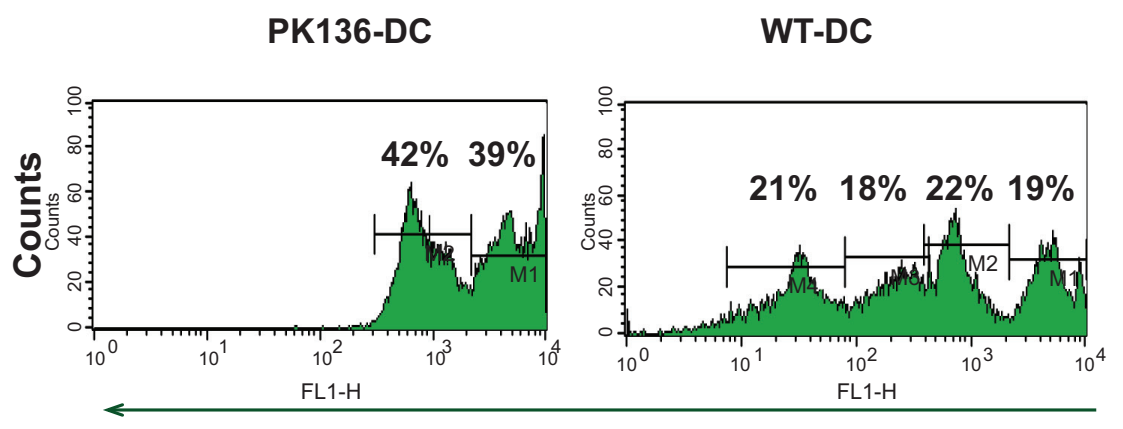

\section{CFSE Dilution of HSV specific T cells}

Fig. (2). DCs in the absence of NK cells are not potent stimulators of $\mathbf{T}$ cells. DCs were isolated from HSV infected (1x $\left.10^{6} \mathrm{pfu}\right) \mathrm{WT}$ C57BL/6 and transgenic PK136 splenocytes via magnetic separation technique. DCs were co-cultured with purified CFSE (5 $\mu$ M) labeled HSV specific CD8+ T from HSV-gB transgenic mice in 0.5:1 ratio. Dilution of CFSE suggests impact of NK cells on the DC's ability to stimulate anti-HSV CD8+ T cells. This experiment was carried out three times with similar outcome. The above result represents one such experiment. The numbers indicate percentage of cells in each division.

address whether or not the activation of DCs in the UnDC/HSVNK resulted from contact with HSV infected NK cells, we compared HSV-DCs alone with UnDC/HSV-NK. HSV-DCs exhibited a significant increased production of IL12 when compared to UnDC/HSV-NK $(p=0.0436)$. No significant difference in phenotypic activation (CD80 or MHCII) was detected when untreated DCs were co-cultured with TLR-L stimulated or HSV-NK cells (data not shown). The concern of carryover virus has been addressed by us in our previous investigation into the mechanism of HSV induced IL-12 production [14]. Briefly, anti-HSV mouse serum was included in some control wells in media to neutralize any free virus and besides an aliquot was also tested for any plaque formation. Based, on these results the chances for free virus particle precluding the results is minimal.

\subsubsection{Effect on NK Cells}

CD69, the early activation marker, was used to measure the NK cells early response to UV-HSV treatment. A significant increase in NK activation (CD3-NK1.1+CD69+) was observed in response to virus exposure. In Fig. (4A), coculturing HSV-DCs with unstimulated NK demonstrated the largest percentage increase of $\mathrm{NK}$ cell activation (UnDC/HSV-NK: 10.3 $\pm 1.6, \quad \mathrm{HSV}-\mathrm{DC} / \mathrm{UnNK}$ : 25.1 \pm 6.2 ; $\mathrm{p} \leq 0.01$ ). In Fig. (4B), analysis of $\mathrm{NK}$ IFN $\gamma$ production highlighted the variations of $\mathrm{NK}$ function in response to stimulation with a dramatic nine fold increase in cytokine production in HSV infected co-cultures when compared to the negative control (Control: $0.30 \pm 0.11$; HSV: 9.39 \pm 1.62 ). HSV-DCs co-culture resulted in increased IFN $\gamma$ production in resting $\mathrm{NK}$ cells compared to direct exposure of the $\mathrm{NK}$ cells suggesting that reduction of the MHC-I on virally infected DCs (ligand for the inhibitory receptor on NK cells) and/or increase in an unknown activating ligand, serves as a stronger inducers of $\mathrm{NK}$ cell function than probable direct virus engagement.

\subsection{Absence of MyD88 Adaptor Protein Decreases NK Cell Function}

The most well known pathway of TLR signaling involves the myeloid differentiation factor 88 (MyD88) adaptor

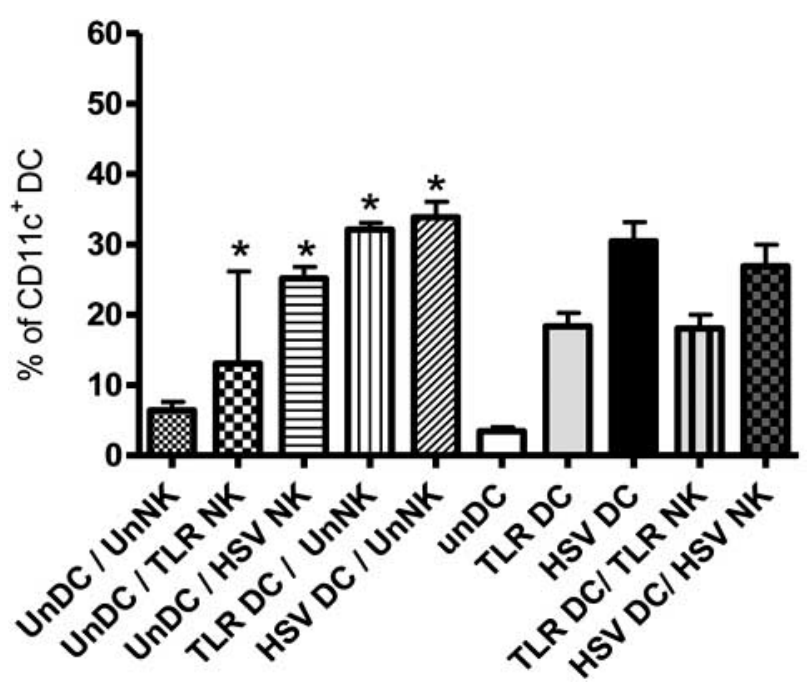

Fig. (3). Concomitant Infection of DCs and NK cells leads to increased DC derived IL-12 Production. One million enriched DCs and NK cells isolated C57BL/6 mice were stimulated with pretitrated synthetic TLR ligands (as described in methods) or UVinactivated HSV for 18 hours. Control groups received mock stimulation. Cell populations were then co-cultured in a $1: 1$ (DC:NK) ratio for 18 hours. IL-12 production was measured using standard intracellular staining protocol. The experiment was repeated three times and the result presented is the average of three separate experiments. The difference between HSV stimulated and TLR stimulated co-cultures were significant. When both DC-NK were exposed to UV-HSV, the expression was significantly higher compared to one of them being stimulated. Significance $(*)$ is $\mathrm{p}<0.05$. Groups labeled with (\#) were significantly different from control group for that particular co-culturing condition. The values shown are mean $\pm \mathrm{SD}$ of three independent experiments.

protein; absence of MyD88 hampers TLR signaling pathways; and mice deficient in MyD88 quickly succumb to lethal encephalitis post HSV-1 infection [15]. We hypothesized that HSV mediated NK cell activation (CD69 expression and IFN $\gamma$ production) will be negatively influenced if MyD88 (and TLR) signaling was negated. To test this, immature DCs and NK cells were generated from $\mathrm{C} 57 \mathrm{BL} / 6$ (WT) and MyD88 $8^{-/-}$bone marrow to yield a 
(A)

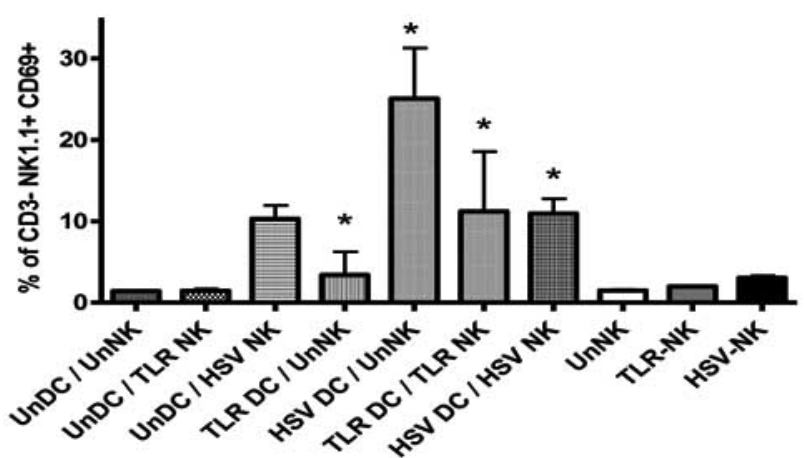

(B)

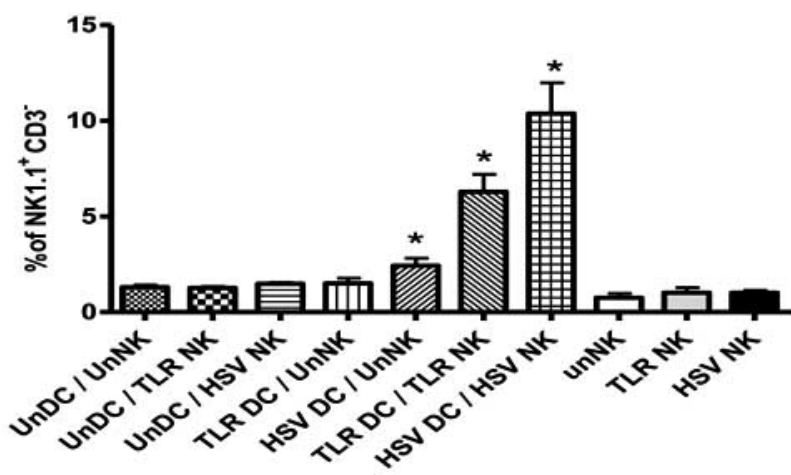

Fig. (4). Increased NK Cell Activation and IFN $\gamma$ production in response to HSV Infection. In a similar co-culture experimental set up as described in Fig. (3), NK cell activation was assessed. Phenotypic changes were examined by measuring CD69, an early activation marker, expression on gated CD3 NK $1.1^{+}$lymphocytes. Cytokine production was determined by intracellular staining for CD3 ${ }^{-} \mathrm{NK} 1.1^{+} \mathrm{IFN} \gamma^{+}$in brefeldin A treated cells. The experiment was repeated multiple times and the figures shows data (mean \pm SD) collected from one of the experiment. ${ }^{*} \mathrm{p}<0.05$.

homogenous population of cells post in vitro expansion. Maturity (DC: CD80, CD86, and MHC-II; NK: CD69) was continually measured via flow cytometry. DCs and NK cells were co-cultured in various combinations and stimulated with UV-HSV or TLR ligands and analyzed for phenotypic and functional changes. Mock stimulation with diluent (endotoxin free buffer) was used as control. Disrupting the TLR signaling (MyD88 ${ }^{-/-} \mathrm{DC}$ ) prevented the up-regulation of co-stimulatory markers on HSV exposed DCs (data not shown). NK cell activation was impacted significantly with CD69 expression being reduced by half (WT-NK: $8.87 \pm 0.49$; MyD88-NK: 4.82 \pm 0.18 ) in the absence of MyD88. Infected Wt DCs were able to induce activation of Wt NK cell (Fig. 5A); however, MyD88 $8^{-/-}$NK cells activation was impinged even in the presence of Wt DCs. Direct activation in HSV-NK cells, CD69 expression, was reduced when compared to NK cells co-cultured with HSVDCs. Further examination into the contribution of DCmediated cytokines to NK cell activation is needed. However, NK cell activation was reduced by $25 \%$ with MyD88 DCs when used in the co-culture. Minimal NK activation was observed in MyD88 deficient NK and DC cocultures (4 fold decrease; WT: $8.87 \pm 0.49 ;$ MyD88:
$2.83 \pm 0.29$ ). Taken together, the results highlight the importance of DC and TLR signaling in HSV mediated activation of NK cells.

(A)

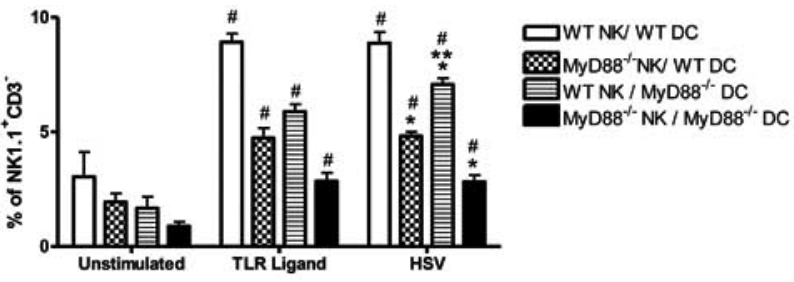

(B)

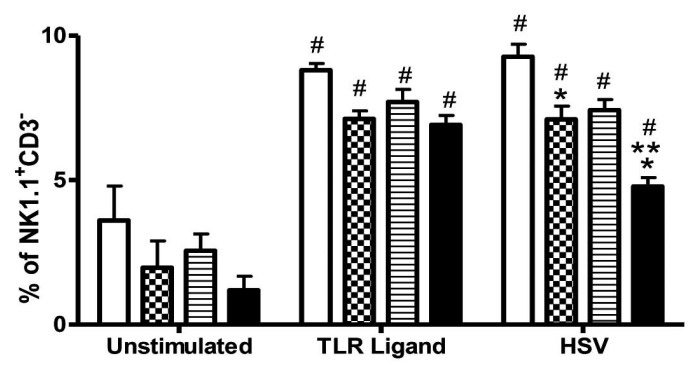

Fig. (5). (A, B) Diminished response to $\mathrm{HSV}$ in $\mathrm{MyD88}^{-/-} \mathrm{NK}$ cells. Dendritic cells and Natural Killer Cells were generated from C57BL/6 and MyD88 ${ }^{-/}$long bone marrow. Cells were supplemented with appropriate growth factors alternate days for 5 days. DCs were stimulated with UV-HSV-KOS strain (MOI of 3 before UV inactivation); stimulated with pre-titrated synthetic TLR ligands (for TLR 2,3,9); or mock stimulated for 18 hours. To examine the impact on NK cells, various groups of stimulated DCs were co-cultured together with NK cells for 18 hours. Cells were labeled with anti-CD3 and anti-NK1.1 to discriminate between NK cells and DC populations. FITC-CD69 was used as an indicator of phenotypic activation of the NK cells (NK1.1+CD3-CD69+). Using the same cell identification markers, the cytokine production of $\mathrm{NK}$ cells was examined by measuring IFN- $\gamma(\mathrm{NK} 1.1+\mathrm{CD} 3-\mathrm{IFN} \gamma+)$ production using standardized intracellular staining procedure. These experiments were carried out three times with similar results and the data shows one such experiment. Data is mean $\pm \mathrm{SD}$ of three independent experiments. Experiments were also carried out in unstimulated cells to serve as a negative control. Significance is $\mathrm{p}<0.05$. *Significance between wild-type DC/wild-type NK and experimental groups. **Significance from TLR ligand stimulation vs HSV stimulation.

Unlike phenotypic activation (CD69 expression), IFN $\gamma$ production seem to be more dependent on the MyD88 adaptor protein presence in the DC population (Fig. 5B). Accordingly, Wt DCs were able to compensate for the lost of MyD88 in NK cells (MyD88 NK/WT DC 7.6 \pm 0.32 vs MyD88 NK/MyD88 DC: $4.8 \pm 0.17)$. The difference was even more pronounced when IFN $\gamma$ was assessed 48 hours after coculture. IFN $\gamma$ production was reduced significantly in the absence of MyD88 in both populations in comparison to other experimental groups. However, the reduction was not as significant as observed with early activation (CD69) of NK cell suggesting that early NK cell activation involves direct virus engagement of TLRs, while continued IFN $\gamma$ production may be mediated by cytokines like IL-12 or IL18 produced by viral PAMPs stimulated DCs. 


\section{DISCUSSION}

In the course of pathogen-induced inflammation, NK cells and DCs are recruited to inflammatory tissues in response to infection [16]. The successive reciprocal interaction results in a potent crosstalk that regulates both the quality and the intensity of innate immune responses [17]. Pathogen-primed NK cells, in the presence of cytokines released by DCs, become activated [13]. At this stage NKs favor DC maturation and also select the most suitable DCs for subsequent migration to lymph nodes for priming of $\mathrm{T}$ cells. Historically the principal function of DCs is the activation of naive $T$ cells [18] and as well as memory $T$ cells [19]. However, DCs are capable of priming natural killer (NK) cells, resulting in enhanced lysis of target cells, IFN- $\gamma$ production and tumor clearance [20]. DC TNF- $\alpha$ production cooperates with IL-12 to influence NK cell IFN- $\gamma$ production [17]. The activated NK cells are capable of enhancing DC maturation and IL-12 production. NK generated IFN- $\gamma$ also increases IL-12 production by DCs thus shaping the ensuing adaptive immune responses towards a $\mathrm{T}$ helper type 1 phenotype [21]. These described interactions are cell contact and TNF- $\alpha$ dependent, and are further controlled by cell ratios, with a low NK: DC ratio (1:5) required for $\mathrm{DC}$ maturation and a high $\mathrm{NK}$ : $\mathrm{DC}$ ratio resulting in inhibition of DC function [22]. We hypothesized that in the case of HSV infection, the activation of NK cells during early stages is initiated through engagement of TLR by viral PAMP with a long term consequence on adaptive immune memory.

Although the engagement of TLRs by HSV is not novel, the role of HSV inducing the interaction NK and DC and subsequent impact the early immunological response remains unclear [23-27]. This study shows the effect of MyD88 signaling on NK:DC reciprocal interaction and its importance for the initiation of an anti-HSV immune response. Using bone marrow derived immature DCs and NK cells from MyD88 ${ }^{-/-}$and WT mice, we addressed the question whether HSV engages TLR on either DCs or NK cells, individually, or concomitantly to induce the initiation of a cooperative relationship. TLR stimulated DCs underwent maturation and up-regulated co-stimulatory molecules (CD80 and CD86) and MHC molecules. HSV perturbs this upregulation and renders the DCs immature [6]. However, HSV DCs significantly enhanced activation and proliferation of resting NK cells compared to direct TLR stimulated NK cells. Concomitant stimulation of both NK cells and DC through MyD88 had the most significant impact on phenotypic changes and functional activation.

HSV-1 activates both TLR2 and TLR9 in a MyD88 dependent manner, suggesting the importance of TLRs in lesion development and host, as well as TLR3 in a MyD88 dependent and -independent mechanism [26-28]. Mansur's studies further suggest that innate resistance to HSV-1 is mediated by MyD88 and may rely on activation of multiple TLRs, indicating a critical role of MyD88 in anti-viral defense [15]. Further studies are needed to examine TRIFdependent signaling not only in the context of NK cells but as well as IFN $\gamma$ production. Multiple studies have examined TRIF signaling in Type I IFN production, yet no evidence has been reported in HSV infection/stimulation model or NK cell activation [29-31].
There are a few hundred TLRs on immature DCs with the number of TLRs expressed by NK cells being unclear [2]. HSV has the potential to engage multiple TLRs on both DCs and NK cells simultaneously or in a multistep process. Sato et al. reported that both TLR2 and 9 can be engaged in sequence within the same DC, a probable mechanism that allows virions to engage TLR2 on the cell surface to induce maturation of endosomes that have taken up the virions to facilitate the ensuing recognition of viral genomic dsDNA by TLR9 [24]. HSV's ability to engage multiple TLRs simultaneously may enable DCs to initiate immune induction more than other cells of the early response, indicating their importance in this process. Our data indicates that HSV PAMPs activate DCs to initiate NK cell activation and initiates a productive interaction.

Our results correlate with numerous other studies in that HSV has not only evolved to infect the professional APC; the virus has acquired efficient means to prevent DC activation following infection and may actually be largely responsible for the phenomenon [6, 32, 33]. Down-regulation of co-stimulatory molecules and MHC-I molecules has been characterized as an HSV immune avoidance mechanism [6]. Consequently, these HSV infected DC are now capable of a productive interaction with resting NK cells according to the "missing-self" theory [34]. Likewise, DCs stimulated with TLR ligand(s) results in the production of key cytokines such as IL-12, IL-15, IL-18 and Type I IFNs, which induce and promote NK cell proliferation, survival, and cytotoxicity (as reviewed in [35]). The cooperative relationship of NK cells with DC (plasmacytoid and myeloid) was effectively compounded suggesting that the role of TLRs in NK cells might be indirect [36]. Yet, no studies have focused on the MyD88 dependent NK cell activation and the ability to augment the NK:DC interaction in the context of HSV.

A MyD88 knock-out system was employed to examine the impact of negating TLR signaling in HSV infection. Bone marrow DCs and NK cells derived from MyD88 knock-out mice were mixed and matched with WT DCs or NK cells and analyzed for the response to HSV or TLR ligands. Ablation of MyD88 mediated signaling resulted in defective production of DC derived IL-12 as also shown by Hou et al. [37]. NK activation and IFN $\gamma$ production was decreased in the absence of MyD88 signaling. However, unlike phenotypic activation, IFN $\gamma$ production seem to be more dependent on the MyD88 adaptor protein presence in the DC population, as WT DCs were able to compensate for the loss of MyD88 in NK cells. IFN $\gamma$ production was reduced significantly in the absence of MyD88 in both populations compared to other combinations suggesting that early NK cell activation involves direct engagement of TLRs by viral PAMPs, while continued IFN $\gamma$ production by NK cells may be mediated by virus activated DC produced cytokines like IL-12 or IL-18. Barr et al. describe the role of DC subpopulations and IL-18 dependent activation of NK cells results following HSV-1 infection [38]. Future studies are needed to examine the contributions of various DCs subpopulations to NK cell immunobiology like has been addressed in context of $\mathrm{T}$ cell priming and activation [39].

However, controversy exists over the precise viral structure or glycoprotein(s) that engage the TLR to initiate 
the cooperation between cells such as NK cells and DCs. HSV infected monocyte derived DC were recently found to respond to viral glycoproteins $\mathrm{gB}, \mathrm{gD}, \mathrm{gH}$ and $\mathrm{gL}$, independently of other viral proteins or nucleic acids leading to up-regulation of costimulatory molecules and HLA-DR. Unexpectedly, all four glycoproteins were necessary and sufficient for the induction of DC maturation [25].

Numerous studies reveal the beneficial impact of administering TLR agonists to enhance the resistance against an infection. Yet, the exact component of HSV or specific glycoprotein(s) that serve as TLR ligands remains to be elucidated. If addressed, therapies could be designed to not necessarily to prevent infection but to decrease the severity of sequelae such as keratitis or encephalitis. In addition, search for HSV proteins that directly engage NK receptors (inhibitory or activating) may yield clues as to the role NK cells play at different stages of infection. Such information will be useful to manipulate NK cells to suit adaptive immune response.

\section{ACKNOWLEDGEMENTS}

We are grateful to Dr. S. Akira (Osaka University, Japan) for generously providing a breeding pair of MyD88 knockout mice. This work was supported in part by NIH ARRA grant PO1 AI083215 (E.A.K.-J.). Special thanks to Dr. Dorothy Yuan (U.T. Southwestern Medical Center, Dallas, TX, USA) for providing the breeding pairs of PK$136 \mathrm{Tg}$ Mice.

\section{REFERENCES}

[1] Jin Y, Fuller L, Ciancio G, et al. Antigen presentation and immune regulatory capacity of immature and mature-enriched antigen presenting (dendritic) cells derived from human bone marrow. Human immunol 2004; 65(2): 93-103.

[2] Akira S, Takeda K, Kaisho T. Toll-like receptors: critical proteins linking innate and acquired immunity. Nat immunol 2001; 2(8): 675-80.

[3] Biron CA. Activation and function of natural killer cell responses during viral infections. Curr Opin Immunol 1997; 9(1): 24-34.

[4] Kassim SH, Rajasagi NK, Zhao X, Chervenak R, Jennings SR. In vivo ablation of CD11c-positive dendritic cells increases susceptibility to herpes simplex virus type 1 infection and diminishes NK and T-cell responses. J Virol 2006; 80(8): 3985-93.

[5] Kassim SH, Rajasagi NK, Ritz BW, et al. Dendritic cells are required for optimal activation of natural killer functions following primary infection with herpes simplex virus type 1. J Virol 2009; 83(7): 3175-86.

[6] Salio M, Cella M, Suter M, Lanzavecchia A. Inhibition of dendritic cell maturation by herpes simplex virus. Eur J Immunol 1999; 29(10): 3245-53.

[7] Nandakumar S, Woolard SN, Yuan D, Rouse BT, Kumaraguru U. Natural killer cells as novel helpers in anti-herpes simplex virus immune response. J Virol 2008; 82(21):10820-31.

[8] Yuan D, Bibi R, Dang T. The role of adjuvant on the regulatory effects of NK cells on B cell responses as revealed by a new model of NK cell deficiency. Int Immunol 2004; 16(5): 707-16.

[9] Wallace ME, Keating R, Heath WR, Carbone FR. The cytotoxic Tcell response to herpes simplex virus type 1 infection of $\mathrm{C} 57 \mathrm{BL} / 6$ mice is almost entirely directed against a single immunodominant determinant. J Virol 1999; 73(9): 7619-26.

[10] Yung YP, Okumura K, Moore MA. Generation of natural killer cell lines from murine long-term bone marrow cultures. J Immunol 1985 ; 134(3): 1462-8.

[11] Kalland T. Generation of natural killer cells from bone marrow precursors in vitro. Immunology 1986; 57(4): 493-8.

[12] Biron CA, Nguyen KB, Pien GC, Cousens LP, Salazar-Mather TP. Natural killer cells in antiviral defense: function and regulation by innate cytokines. Annu Rev Immunol 1999; 17: 189-220.
[13] Moretta A. Natural killer cells and dendritic cells: rendezvous in abused tissues. Nat Rev 2002; 2(12): 957-64.

[14] Kumaraguru U, Rouse BT. The IL-12 response to herpes simplex virus is mainly a paracrine response of reactive inflammatory cells. J Leukoc Biol 2002; 72(3): 564-70.

[15] Mansur DS, Kroon EG, Nogueira ML, et al. Lethal encephalitis in myeloid differentiation factor 88-deficient mice infected with herpes simplex virus 1. Am J Pathol 2005; 166(5): 1419-26.

[16] Della Chiesa M, Sivori S, Castriconi R, Marcenaro E, Moretta A. Pathogen-induced private conversations between natural killer and dendritic cells. Trends Microbiol 2005; 13(3): 128-36.

[17] Zitvogel L. Dendritic and natural killer cells cooperate in the control/switch of innate immunity. J Exp Med 2002; 195(3): F9-14.

[18] Nussenzweig MC, Steinman RM. Contribution of dendritic cells to stimulation of the murine syngeneic mixed leukocyte reaction. $\mathrm{J}$ Exp Med 1980; 151(5): 1196-212.

[19] Zammit DJ, Cauley LS, Pham QM, Lefrancois L. Dendritic cells maximize the memory CD8 $\mathrm{T}$ cell response to infection. Immunity 2005; 22(5): 561-70

[20] Fernandez NC, Lozier A, Flament C, et al. Dendritic cells directly trigger NK cell functions: cross-talk relevant in innate anti-tumor immune responses in vivo. Nat Med 1999; 5(4): 405-11.

[21] Mailliard RB, Son Y-I, Redlinger R, et al. Dendritic cells mediate NK cell help for Th1 and CTL responses: two-signal requirement for the induction of NK cell helper function. J Immunol 2003; 171(5): 2366-73.

[22] Piccioli D, Sbrana S, Melandri E, Valiante NM. Contact-dependent stimulation and inhibition of dendritic cells by natural killer cells. J Exp Med 2002; 195(3): 335-41.

[23] Kurt-Jones EA, Belko J, Yu C, et al. The role of toll-like receptors in herpes simplex infection in neonates. J Infect Dis 2005; 191(5): 746-8.

[24] Sato A, Linehan MM, Iwasaki A. Dual recognition of herpes simplex viruses by TLR2 and TLR9 in dendritic cells. Proc Natl Acad Sci USA 2006; 103(46): 17343-8.

[25] Reske A, Pollara G, Krummenacher C, Katz DR, Chain BM. Glycoprotein-dependent and TLR2-independent innate immune recognition of herpes simplex virus-1 by dendritic cells. J Immunol 2008; 180(11): 7525-36.

[26] Kurt-Jones EA, Chan M, Zhou S, et al. Herpes simplex virus 1 interaction with Toll-like receptor 2 contributes to lethal encephalitis. Proc Natl Acad Sci USA 2004; 101(5): 1315-20.

[27] Krug A, Luker GD, Barchet W, Leib DA, Akira S, Colonna M. Herpes simplex virus type 1 activates murine natural interferonproducing cells through toll-like receptor 9. Blood 2004; 103(4): 1433-7.

[28] Kurt-Jones EA, Popova L, Kwinn L, et al. Pattern recognition receptors TLR4 and CD14 mediate response to respiratory syncytial virus. Nat immunol 2000; 1(5): 398-401.

[29] Han KJ, Su X, Xu LG, Bin LH, Zhang J, Shu HB. Mechanisms of the TRIF-induced interferon-stimulated response element and NFkappaB activation and apoptosis pathways. J Biol Chem 2004; 279(15): 15652-61.

[30] Watts C. Location, location, location: identifying the neighborhoods of LPS signaling. Nat immunol 2008; 9(4): 343-5.

[31] Chang EY, Guo B, Doyle SE, Cheng G. Cutting edge: involvement of the type I IFN production and signaling pathway in lipopolysaccharide-induced IL-10 production. J Immunol 2007; 178(11): 6705-9.

[32] Mikloska Z, Bosnjak L, Cunningham AL. Immature monocytederived dendritic cells are productively infected with herpes simplex virus type 1. J Virol 2001; 75(13): 5958-64.

[33] Bosnjak L, Miranda-Saksena M, Koelle DM, Boadle RA, Jones CA, Cunningham AL. Herpes simplex virus infection of human dendritic cells induces apoptosis and allows cross-presentation via uninfected dendritic cells. J Immunol 2005; 174(4): 2220-7.

[34] Kiessling R, Klein E, Wigzell H. "Natural" killer cells in the mouse. I. Cytotoxic cells with specificity for mouse Moloney leukemia cells. Specificity and distribution according to genotype. Eur J Immunol 1975; 5(2): 112-7.

[35] Iwasaki A, Medzhitov R. Toll-like receptor control of the adaptive immune responses. Nat immunol 2004; 5(10): 987-95.

[36] Gerosa F, Baldani-Guerra B, Nisii C, Marchesini V, Carra G, Trinchieri G. Reciprocal activating interaction between natural killer cells and dendritic cells. J Exp Med 2002; 195(3): 327-33. 
[37] Hou B, Reizis B, Defranco AL. Toll-like receptors activate innate and adaptive immunity by using dendritic cell-intrinsic and extrinsic mechanisms. Immunity 2008; 29(2): 272-82.

[38] Barr DP, Belz GT, Reading PC, et al. A role for plasmacytoid dendritic cells in the rapid IL-18-dependent activation of NK cells following HSV-1 infection. Eur J Immunol 2007; 37(5): 1334-42.
[39] Jirmo AC, Nagel CH, Bohnen C, Sodeik B, Behrens GM. Contribution of direct and cross-presentation to CTL immunity against herpes simplex virus 1 . J Immunol 2009; 182(1): 283-92.

(C) Woolard et al.; Licensee Bentham Open.

This is an open access article licensed under the terms of the Creative Commons Attribution Non-Commercial License (http://creativecommons.org/licenses/by$\mathrm{nc} / 3.0 /$ ) which permits unrestricted, non-commercial use, distribution and reproduction in any medium, provided the work is properly cited. 